\title{
Review Article \\ The Protective Role of Probiotics against Colorectal Cancer
}

\author{
Sujuan Ding $\mathbb{D}$, Chao Hu, Jun Fang $\mathbb{D}$, and Gang Liu $\mathbb{D}$ \\ College of Bioscience and Biotechnology, Hunan Agricultural University, Hunan Provincial Engineering Research Center of Applied \\ Microbial Resources Development for Livestock and Poultry, Changsha, Hunan 410128, China
}

Correspondence should be addressed to Jun Fang; fangjun1973@hunau.edu.cn and Gang Liu; gangle.liu@gmail.com

Received 29 September 2020; Revised 2 November 2020; Accepted 26 November 2020; Published 9 December 2020

Academic Editor: Qiang Tong

Copyright (C) 2020 Sujuan Ding et al. This is an open access article distributed under the Creative Commons Attribution License, which permits unrestricted use, distribution, and reproduction in any medium, provided the original work is properly cited.

Colorectal cancer (CRC) is the fourth leading cause of cancer-related deaths worldwide and a major global public health problem. With the rapid development of the economy, the incidence of CRC has increased linearly. Accumulating evidence indicates that changes in the gut microenvironment, such as undesirable changes in the microbiota composition, provide favorable conditions for intestinal inflammation and shaping the tumor growth environment, whereas administration of certain probiotics can reverse this situation to a certain extent. This review summarizes the roles of probiotics in the regulation of CRC, such as enhancing the immune barrier, regulating the intestinal immune state, inhibiting pathogenic enzyme activity, regulating CRC cell proliferation and apoptosis, regulating redox homeostasis, and reprograming intestinal microbial composition. Abundant studies have provided a theoretical foundation for the roles of probiotics in CRC prevention and treatment, but their mechanisms of action remain to be investigated, and further clinical trials are warranted for the application of probiotics in the target population.

\section{Introduction}

The global incidence of CRC is very high and continues to increase every year. Data show that CRC accounts for approximately $9 \%$ of all cancer-related deaths and is the third leading cause of death in women after breast cancer and the second leading cause of death in men after lung and prostate cancers $[1,2]$. Despite advances in screening and early diagnosis of CRC, CRC remains the second leading cause of cancer-related deaths. Therefore, more research attention to CRC prevention, treatment, and prognosis is crucial.

Recent evidence has demonstrated that probiotics may contribute to the treatment of CRC [3]. According to the definition established in 2002 by the Food and Agriculture Organization of the United Nations (FAO) and the World Health Organization (WHO), probiotics are "live microorganisms which when administered in adequate amounts confer a health benefit on the host" [4]. Several studies have highlighted the critical role of probiotics in regulating intestinal disorders, such as diarrhea [5], inflammatory bowel disease [6], irritable bowel syndrome [7], Helicobacter pylori infection [8], and lactose intolerance [9]. Probiotics can also inhibit the development of CRC by modifying the intestinal microbial composition, intestinal epithelial system, and intestinal immune responses. Akkermansia muciniphila (AKK), an intestinal symbiotic bacterium living in the mucosal layer, has been shown to exhibit a high antitumor efficacy with favorable clinical outcomes $[10,11]$. One study demonstrated that AKK initiates an antitumor immune response by activating the Toll-like receptor signaling pathway through its outer membrane protein Amuc. Meanwhile, it is found that the administration of AKK together with interleukin(IL-) 2 protects the intestinal barrier function, suggesting a new therapeutic strategy for CRC [12].

\section{Interaction between Probiotics and the Host}

Probiotics used in foods are safe for human consumption, with most being certified as Generally Regarded as Safe (GRAS) by the U.S. FDA or as Qualified Presumption of Safety (QPS) by the E.U. EFSA [13]. Recent studies based on animal models and clinical interventions have demonstrated the critical role of probiotics in the prevention and treatment of several human diseases [14]. The interplay between probiotics and the human gastrointestinal tract (GIT), comprising the mucus layer, epithelial layer, and 
gut-associated lymphoid tissue, influences the disease process in the human host [15]. The mucosal layer of the intestinal tract comprises a loose outer sublayer of gel-forming mucins and a dense inner sublayer of mucins. The outer sublayer is relatively abundant with bacteria, antimicrobial peptides, and immunoglobulin, whereas the inner sublayer has few or no microbes [16, 17]. The secondary interaction between probiotics and the intestinal tract occurs in the intestinal epithelial layer containing different cell subgroups and spanning across the entire intestinal cavity. The main functions of this layer are absorption of nutrients, secretion of mucin, and release of antimicrobial molecules such as defensin and lysozyme [18]. Bacteria affect the intestinal epithelial barrier function through pattern recognition receptors [19]. Probiotics interact with host intestinal epithelial cells (IECs) by adhering to the intestinal wall and stimulating the production of mucus, thereby enhancing the intestinal barrier [20]. Through such interaction, probiotics compete with pathogenic bacteria for niche occupancy [21], prevent pathogenic bacteria from growing and proliferating in the intestine by competing with them for nutrition and energy $[22,23]$, and reduce intestinal $\mathrm{pH}$ by fermenting dietary fiber to produce short-chain fatty acids (SCFAs) [24].

\section{Colorectal Cancer}

CRC causes nearly 700,000 deaths every year, making it the most fatal cancer in the world after lung cancer, liver cancer, and gastric cancer [25]. Unhealthy eating habits, especially frequent consumption of low-fiber and high-fat foods characteristic of the Western diet, are crucial factors in the development of intestinal disorders [26], which suggests that the prevalence of the Western diet and lifestyle also increases the incidence of CRC. CRC is a slow-developing disease, and survival rates have improved in recent decades owing to the improvements in preventive cancer screening, which allows early detection. Screening thus remains the mainstay for CRC prevention [27]. CRC is believed to be associated with aging, and the majority of people who undergo regular screening for CRC are older than 50 years; this underestimates the likelihood of CRC in younger patients, even when they present with abdominal pain and bloody stools [28].

Further advancements in the prevention and treatment of CRC warrant a complete understanding of the normal biology of the colon and the pathogenesis of CRC. The basic unit of the colon includes crypts and luminal surfaces. When the intestine is in a state of homeostasis, each colon crypt contains 14-16 pluripotent stem cells marked with the transmembrane protein leucine-rich repeat-containing $G$ proteincoupled receptor 5 (LGR5). These stem cells can produce all differentiated cell types in the colon cavity $[29,30]$. LGR5 ${ }^{+}$ stem cells can produce rapidly proliferating transitamplifying (TA) cells, which account for approximately two-thirds of the crypts. TA cells mainly differentiate into four cell types, namely, absorbable IECs, goblet cells, cluster cells, and intestinal endocrine cells, which are renewed approximately once a week [31]. The main transcription target of the Wnt pathway in intestinal crypt stem cells is the serpentine transmembrane receptor LGR5, which inhibits the expression of the oncogene $M y c$ and of the basic helixloop-helix (bHLH) transcription factor achaete-scute like 2 (ASCL2), which is associated with stem cell self-renewal [32]. Mutations in the adenomatous polyposis coli (APC) gene are the potential cause of familial adenomatous polyposis, known as hereditary colon cancer syndrome [33, 34]. APC loss is also the major driver of Wnt signaling in CRC [35]. Evidence indicates that different $A P C$ mutations result in different levels of Wnt signaling pathway activity, which is related to the typical tumor location in the large intestine $[36,37]$.

\section{Gut Microbiota}

The human gut microbiota is a rich, diverse, and complex microbial community composed of fungi, bacteria, archaea, viruses, bacteriophages, and protozoa living in a symbiotic relationship with the human host [38]. The composition and activity of the gut microbiota is a hot topic in the cross-research field of human microbiology and health, and it is directly related to the study of probiotics [15]. The commensal bacteria form a tight and complex interaction network with their hosts and are involved in protecting the gut from harmful substances [39]. Metagenomic evidence suggests that the gene set of different gut microbial species pools and the functional prediction of the community are the same and similar, respectively, among individuals. However, the composition and function of the gut microbiota vary with diet, location, sex, age, and race $[40,41]$. Diet is the main regulator of the intestinal microbial function. In general, the ratio of the phyla Firmicutes/Bacteroidetes is higher in individuals following a Western-style diet, whereas the abundance of the genus Prevotella, belonging to the Bacteroidetes phylum, is higher in individuals following a subsistence diet [42-45]. In healthy individuals, more than $90 \%$ of the ingested diet is absorbed by the small intestine, whereas the complex carbohydrates that pass undigested from the small intestine, such as fiber, protein residues, and primary bile acids secreted by the body in response to fat intake, are digested in the colon [46]. These components of the diet influence the composition and function of the gut microbiota. Saccharolytic fermentation of complex carbohydrates by the colonic bacteria produces SCFAs, with acetic, propionic, and butyric acids (in a molar ratio of $3: 1: 1$ ) accounting for approximately $90 \%-95 \%$ of colonic SCFAs $[47,48]$. Butyrate regulates mucosal inflammation and antitumor activity by participating in intestinal microbial balance, proliferation inhibition, immune regulation, and epigenetic regulation [49].

The gut microbiota is composed of more than 1,000 bacterial species, including beneficial and pathogenic microbes, and is dominated by Firmicutes and Bacteroidetes. In healthy individuals, the beneficial microbes surpass the pathogenic microbes and inhibit their excessive growth [50]. The gut microbiota can thus be considered as an "organ" that performs significant roles, including the utilization of complex dietary constituents, anabolism of various important compounds, regulation of immune function, and maintenance of intestinal barrier integrity [51]. Hence, the role of the gut 
microbiota in the pathogenesis of intestinal disorders cannot be underestimated, and its role in the pathogenesis of CRC has received much attention in recent years [52]. Whether microbiota dysbiosis is the cause or result of CRC is still unknown, which remains a foundational issue in understanding CRC [25]. The occurrence of CRC is usually closely related to the mucosal microbes near the site of tumorigenesis [53-55]. The main bacterial species that influence the development of CRC are not yet completely clear, but the available evidence suggests that the abundances of Fusobacterium nucleatum (Fn), Escherichia coli, Helicobacter pylori, and Bacteroides fragilis are closely associated with CRC [56]. It is also suggested that a decrease in bacterial diversity is related to the occurrence of tumors, but its role in tumorigenesis remains to be confirmed in further studies [57].

\section{Mechanism Underlying the Role of Probiotics in the Regulation of CRC}

Research on bioactive components and gut microbes has revealed that probiotics may play an important role in cancer prevention and treatment in addition to regulating the homeostasis and immune state of the intestinal epithelial system [58]. Multiple mechanisms have been hypothesized for the CRC-preventive and therapeutic effects of probiotics. For example, at the level of intestinal ecology, probiotics may reduce the number of pathogenic bacteria in the gut by competing with the pathogenic bacteria for intestinal niche occupancy or reduce the level of carcinogens [59]. In addition, SCFAs produced by microbial metabolism could stimulate the proliferation and differentiation of intestinal cells in the large and small intestines [60]. For instance, intestinal acetic acid produced by Propionibacterium can trigger the release of cathepsin D into the cytosol of cancer cells by increasing the permeability of their lysosomal membrane, thereby protecting the cells from apoptosis [61]. In this section, we focus on the various roles of probiotics, including enhancing the intestinal mucosal barrier, reducing intestinal inflammation, inhibiting the activity of pathogenic bacteria, regulating redox homeostasis, and reprogramming the composition of microorganisms, in the regulation of CRC (Figure 1).

5.1. Enhancing the Intestinal Mucosal Barrier. The complete intestinal mucosal barrier includes physical, chemical, biological, and immune barriers. In a healthy state, the intestinal barrier can protect the gut from toxins and pathogens [62]. Probiotics stimulate mucus secretion by IECs, which functions as a barrier between the mucosa and microorganisms that prevents the translocation of bacteria and toxins and also inhibits the adhesion and invasion of pathogenic bacteria in IECs [63]. Probiotics enhance the intestinal barrier by regulating the expression of tight junction proteins, such as claudin-1 and occludin, and stimulating intestinal cells to suppress inflammation and accelerate epithelial cell remodeling by promoting mucin secretion [64-66]. Occludin is a transmembrane tight junction protein that forms the mechanical barrier of epithelial cells, and the level of occludin is a functional indicator of the intestinal mechanical barrier
[67]. Bifidobacterium infantis and Lactobacillus acidophilus were found to protect intestinal permeability by regulating the expression of occludin and claudin-1 proteins and protecting the activation of nuclear factor kappa-B (NF- $\kappa \mathrm{B}$ ) induced by IL- $1 \beta$ in Caco-2 cells [68]. Lactobacillus plantarum ZLP001 reversed the decrease in claudin-1 and occludin protein levels induced by enterotoxigenic E. coli and decreased the levels of the inflammatory cytokines IL-6, IL8 , and tumor necrosis factor alpha (TNF- $\alpha$ ) [69]. Mucin-2 glycoprotein (MUC2) formed by goblet cells in the form of a disulfide cross-linked network is the main component of colonic mucus [70]. Muc2 gene inactivation in mice has been shown to increase close contact between bacteria and IECs, leading to inflammation and eventually colon cancer [71]. SCFAs produced by microbes through fermentation of complex carbohydrates can enhance barrier function by $G$ protein-coupled receptor-mediated sensitization of the IEC inflammasome and reducing the oxygen concentration of IECs to induce hypoxia-inducible factors [56].

5.2. Reducing Intestinal Inflammation. Immunotherapy involves the stimulation of innate immunity and the subsequent activation of antitumor immune responses [72]. Evidence suggests that the mechanism of inflammation is a driver of tumor maturation and that inflammation is closely associated with the risk of CRC [73]. The gut microbiota plays an important role in the formation of an inflammatory microenvironment, and the occurrence of inflammation in turn affects the composition of the gut microbiota. Intestinal tumorigenesis is driven by inflammation, microbes, and immunity [74]. Probiotics contribute to the normal functioning of the immune system and affect the host immune status by participating in the differentiation of immune cells and stimulating the production of anti-inflammatory substances, antioxidants, and antitumor components $[66,75,76]$. The colonic immune system contains many types of immune cells, with macrophage being one of the most abundant immune cell types [77-79]. A possible mechanism by which probiotics improve the stability of the colonic environment is by acting on the colonic macrophages [80]. Macrophages perform probiotic phagocytosis in a strain-dependent manner and prevent deep tissue destruction after infection by secreting anti-inflammatory mediators [60]. Evidence has revealed that the interaction between probiotics and Tolllike receptors expressed on IECs leads to the production of TNF in the cells, which inhibits NF- $\kappa$ B in macrophages and stimulates the production of IL- 8 required for neutrophil production [81]. A study showed that heat-killed Enterococcus faecalis could reduce caspase- 1 activity and IL- $1 \beta$ maturity, thereby achieving consistent activation of the NLRP3 inflammasome in macrophages [82]. Furthermore, SCFAs produced by dietary fiber fermentation are not only the main energy source for IECs but also the regulator of the intestinal immune response [83]. Mechanistically, the induction of a tumor phenotype may be due to the proliferation of colon epithelial cells induced by butyrate. However, butyric acid and its receptor GPR109A can also inhibit colitis and tumorigenesis, indicating that butyrate has anticancer potential [84]. 


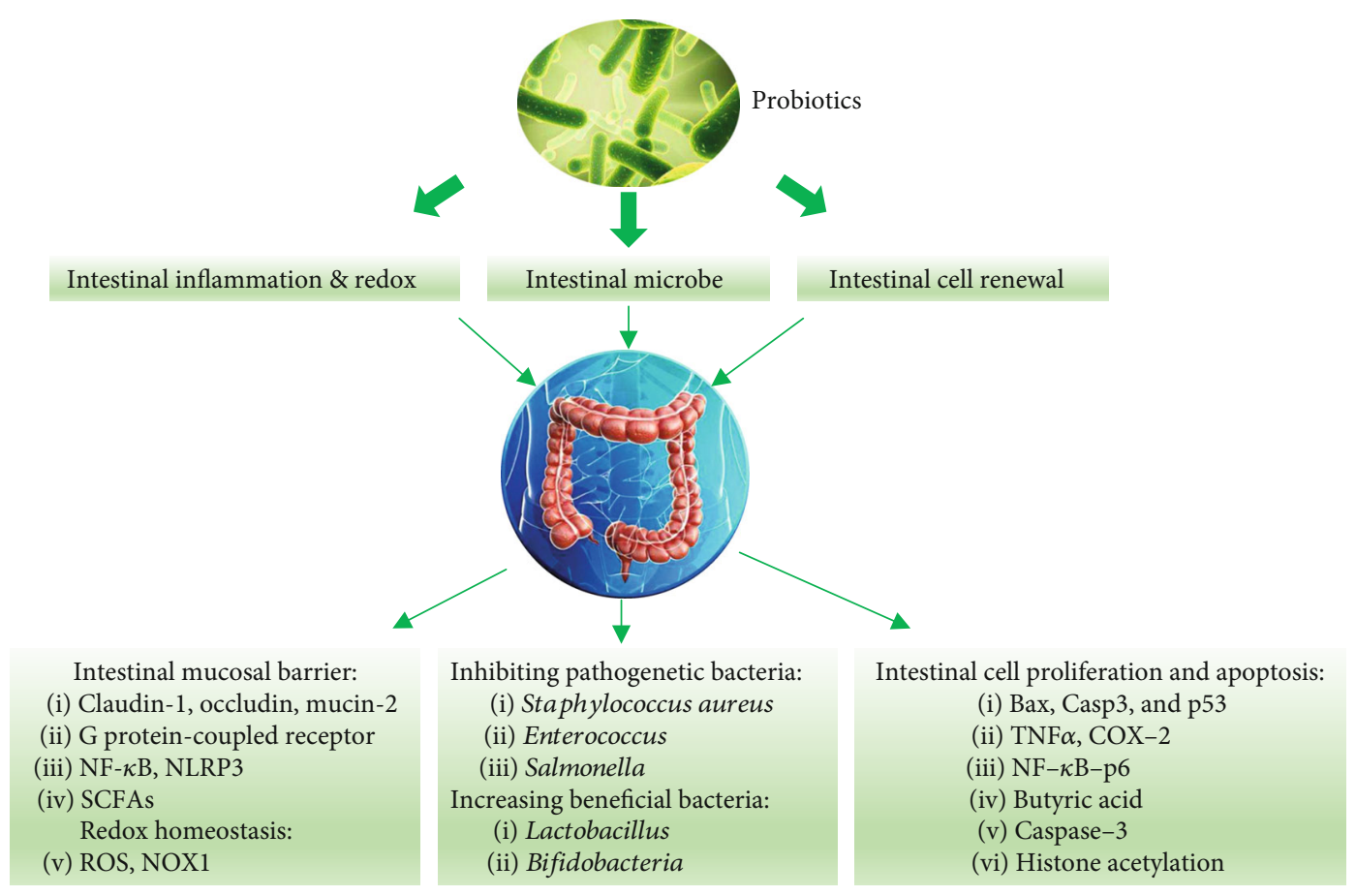

FIGURE 1: The various roles of probiotics in colorectal cancer prevention and treatment.

5.3. Regulating the Generation of Reactive Oxygen Species. Oxidative stress plays a vital role in the occurrence of CRC [85]. Reactive oxygen species (ROS) are by-products of normal cell metabolism in the GIT. The control of redox homeostasis by the intestinal epithelium, that is, the balance between antioxidation and oxidative stress, is a vital factor affecting intestinal functions such as digestion and absorption of nutrients, immune response, stem cell proliferation, and apoptosis of apical enterocyte [86-88]. ROS and its oxidation products may damage the antioxidant system of intestinal tissues and destroy the normal function of the intestine, potentially leading to intestinal mucosal hyperplasia [89-91]. DNA mutations caused by ROS are thought to be involved in the early inflammatory process of CRC development [92, 93]. Nicotinamide adenine dinucleotide phosphate oxidase (NOX), expressed on the surface of inflammatory phagocytes such as neutrophils and phagocytes, participates in ROS generation. It is also involved in the proliferation and invasion of epithelial tumor cells. ROS produced by NOX1 can in turn trigger angiogenesis in the epithelial tumor cells by inducing angiogenic factors, thus promoting their vascularization and proliferation $[94,95]$. Gut microbial dysbiosis caused by the mucosa-associated immune system may promote leukocyte-induced inflammation and oxidative overreaction, consequently aggravating intestinal mucosal injury [96]. Of the colonic commensal bacteria considered to play a crucial role in CRC development, enterotoxigenic Bacteroides fragilis (ETBF) is suggested to cause inflammatory diarrhea by secreting toxins [97]. B. fragilis toxin promotes the production of ROS in IECs and dendritic cells [98, 99]. A study showed that commensal bacterial rapidly produced ROS on IECs both in vitro and in vivo and caused oxidative inactivation of the catalytic cysteine residue of $\mathrm{Ubc12}$, resulting in the suppression of the cullin-1 ubiquitination and the consequent inhibition of NF- $\kappa \mathrm{B}$ and $\beta$-catenin signaling pathways [100].

Research on the role of the gut microbiota in regulating gastrointestinal redox homeostasis is still in its infancy. However, some preliminary data have uncovered the relationship between the microbiota and redox status, which plays an important role in the regulation of gastrointestinal health. Evidence suggests that the hosts' ROS is associated with the balance of the gut microbial composition; for instance, the oxidation state of the host is negatively correlated with the abundance of Lactobacillus and Bifidobacterium and positively correlated with that of E. coli [101]. Findings from mouse models have indicated that a high abundance of Bacteroidetes in the colon controls pathogen loads by inducing proinflammatory and prooxidative reactions, which play a key role in preventing intestinal infections [102]. The results of a study in a mouse model of CRC induced by azoxymethane showed that the structure of the intestinal microbiota was regulated by Clostridium butyricum administered by gavage, which involved a reduction of the ratio of Firmicutes/Bacteroidetes, an increase in the relative abundance of probiotics, an increase in tumor cell apoptosis, inhibition of the NF- $\kappa$ B pathway and IL- 6 levels, and a reduction in CRC incidence [103]. In one study, the supernatants of Musa paradisiaca inflorescence fermented with Lactobacillus casei and Bifidobacterium bifidum were found to induce DNA damage, promote ROS generation, and initiate the apoptosis signaling pathway in HT-29 colon cancer cells [104]. Another study showed that Lactobacillus paracasei subsp. paracasei M5L suppressed HT-29 cell proliferation and could promote HT-29 cell apoptosis through ROS production and calreticulin translocation [105]. Moreover, Lactobacillus can 
exert anticancer effects by producing antioxidants such as glutathione, superoxide dismutase, and catalase, suppressing inflammation and tumor size, and inhibiting the expression of tumor-specific proteins and polyamine components. However, the mechanism of the anticancer effect of Lactobacillus in relation to CRC needs to be investigated further [106-108].

\subsection{Inhibiting the Enzyme Activity of Pathogenic Bacteria.} Endogenous toxic compounds, such as N-nitroso, cresol, aglycones, and phenols, promote the development of CRC by participating in antiapoptotic pathways in the intestine. The carcinogenic effects of endogenous toxic and genotoxic compounds in the intestinal microenvironment may be further influenced by pathogenic bacterial enzymes such as 7$\beta$-dehydroxylase, nitroreductase, $\beta$-glucuronidase, $\beta$-glucosidase, and azoreductase $[109,110]$. For example, pathogenic bacteria such as Staphylococcus aureus, Enterococcus, and Salmonella synthesize azoreductase, which metabolizes dyes and drugs to generate toxic aromatic amines [111]. Polyketide synthase (pks) islands present in some strains of E. coli encode the genotoxin colicin, which can induce singlestranded DNA breaks [112]. Furthermore, the DNA damage response signaling pathway activated in infected cells tends to increase the mutation rate [113]. Enterotoxigenic B. fragilis has been reported to participate in CRC initiation by producing a toxin [114]. Nevertheless, studies have shown that probiotic supplementation may suppress the activity of bacterial enzymes $[115,116]$. For example, Lactobacillus could suppress the dehydrogenation of L. rhamnosus GG (LGG) and reduce the level of primary bile acid by reducing the activity of $\beta$-glucuronidase [117]. Animal model studies have shown that yogurt starter bacteria could reduce the activity of bacterial enzymes, which may be the mechanism underlying the CRC-preventive effects of probiotics [118]. However, in healthy subjects, L. acidophilus A1, L. plantarum 299V, and L. rhamnosus DR20 could not decrease glucuronidase activity $[119,120]$.

5.5. Regulating the Proliferation and Apoptotic Responses of CRC Cells. Apoptosis plays a key role in regulating the number of cells by balancing cell renewal and eliminating mutant cells, which is one of the main mechanisms of tumor cell death in CRC. The decrease in apoptosis is an important disease event and is accompanied by disruption of cell proliferation regulation [121]. Therefore, apoptotic pathways are a promising target for disease prevention and treatment to manage cell survival and death through apoptosis regulation. Accumulating evidence has highlighted the critical role of probiotics in the regulation of cell proliferation and apoptosis, which may thus be a vital therapeutic and preventive measure against CRC [122]. In rat models, LGG decreased the incidence and size of dimethylhydrazine-induced tumors while inhibiting the expression of inflammatory proteins, namely, TNF- $\alpha$, COX-2, and NF- $\kappa \mathrm{B}-\mathrm{p} 6$, reducing the expression of the antiapoptotic protein $\mathrm{Bcl}-2$, and increasing the expression of the proapoptotic proteins Bax, Casp3, and p53, suggesting that LGG has the potential to prevent colon cancer [123]. In another study, L. plantarum DY-1 showed a strong antiproliferative activity in an HT-29 cell model that involved retarding the development of the cell cycle from G0G1 phase to G2-M phase and induction of cell apoptosis possibly via caspase-3, indicating that $L$. plantarum DY-1 has antitumor potential [124]. In addition, SCFAs reduce cancer risk by reducing tumor growth and activating apoptosis cascades via hyperacetylation of histones [125]. Propionibacterium freudenreichii, a probiotic in the human gut microbiota, has been found to suppress colorectal adenocarcinoma cells via SCFA-mediated apoptosis [126]. Butyric acid was found to prevent CRC by regulating the cell cycle, differentiation, and apoptosis of colon cancer cell lines [127-129].

5.6. Reprogramming the Composition of Gut Microbes. The ultimate goal of probiotic intervention is to exert regulatory effects, including immune regulation, immune barrier strengthening, and regulation of the gut microbial composition, against certain disorders [15]. Changes in the gut microbial composition are inextricably linked to the development of CRC. Substantial evidence from animal model studies suggests that probiotics, such as Lactobacillus and Bifidobacterium, have significant effects on intestinal microbial composition $[130,131]$. The colon is teeming with microbes, and this large population is mostly benign, but some are pathogenic bacteria, and the increase in the abundance of these pathogens in the colon is associated with acute or chronic conditions, such as obesity, inflammatory bowel disease, and CRC [132]. E. coli is an intestinal symbiotic bacterium, and certain strains of it can promote intestinal inflammation leading to the production of colicin, a potential carcinogen [133]. Pathogenic E. coli exists in CRC tissues and is thus used as a marker in tumor staging and prognosis [134]. Furthermore, as noted earlier, E. coli containing pks islands, which encode colibactin, can induce singlestranded DNA breaks, and thus, changes in the E. coli gene set influence the phenotype of the disease [112, 135]. Compared with mice injected with $E$. coli, those injected with Bacillus polyfermenticus showed reduced tumor size, while HT-29 cells injected with $B$. polyfermenticus showed reduced expression of ErbB2 and ErbB3 at the protein and mRNA levels [136, 137]. Intestinal pathogenic microbes such as Bacteroides and Clostridium are associated with the pathogenesis of CRC [138]. A double-blind test of synbiotics (LGG, Bifidobacterium lactis $\mathrm{Bb} 12$, and oligofructose) in 37 patients with $\mathrm{CRC}$ and 43 colonic polypectomy patients demonstrated that the abundance of Lactobacillus and Bifidobacterium increased, whereas that of Clostridium perfringens decreased in CRC patients, and synbiotic intervention inhibited the colorectal cell proliferation ability and colon cell necrosis ability and improved epithelial cell barrier function in colonic polypectomy patients [139].

\section{Perspectives}

Although certain bacterial species are classified as probiotics due to their benefits to the host health, changes in host health status require the regulation of specific probiotic bacteria rather than the probiotic community in the gut. Substantial research has explored the role of probiotics in the prevention, 
treatment, and prognosis of CRC. Such dedicated research has revealed a variety of regulatory roles of probiotics, such as enhancing the immune barrier, regulating the intestinal immune state, inhibiting pathogenic enzyme activity, regulating CRC cell proliferation and apoptosis, and regulating the intestinal microbial composition. Although the evidence from clinical or animal model experiments has provided a theoretical foundation for the application of probiotics, evidence from clinical trials on the benefits of probiotics in the prevention and treatment of CRC is lacking. Therefore, further clinical trials are warranted to explore the mechanisms of probiotics in the regulation of CRC. In addition, it remains unknown whether gut microbial dysbiosis is the cause or result of CRC. To address this knowledge gap, further studies on the interactions between probiotics and intestinal microorganisms in CRC development are warranted. Meanwhile, although the gut microbiota contains fungi and viruses in addition to bacteria, there is little evidence supporting the role of fungi and viruses in the gut microbial dysbiosis leading to CRC development.

\section{Conflicts of Interest}

The authors declare there is no conflict of interest to report.

\section{Authors' Contributions}

Writing (original draft preparation) was handled by S.D. and C.H.; writing (review and editing) was made by J.F. and G.L. All the authors contributed to manuscript revision and read and approved the submitted version.

\section{Acknowledgments}

This study was supported by the National Natural Science Foundation of China (Nos. 31672457 and 31772642), Hunan Provincial Science and Technology Department (2019TP2004), Double First-Class Construction Project of Hunan Agricultural University (SYL201802003), China Postdoctoral Science Foundation (2018M632963 and 2019T120705), and Doctoral dissertation Cultivation Fund of Hunan Agricultural University (YB2018007).

\section{References}

[1] J. Ferlay, I. Soerjomataram, R. Dikshit et al., "Cancer incidence and mortality worldwide: sources, methods and major patterns in GLOBOCAN 2012," International Journal of Cancer, vol. 136, no. 5, pp. E359-E386, 2015.

[2] D. E. Beck, "The importance of colorectal cancer screening," The Ochsner Journal, vol. 15, no. 1, pp. 11-12, 2015.

[3] R. Fletcher, Y. J. Wang, R. E. Schoen, O. J. Finn, J. Yu, and L. Zhang, "Colorectal cancer prevention: immune modulation taking the stage," Biochimica Et Biophysica Acta. Reviews on Cancer, vol. 1869, no. 2, pp. 138-148, 2018.

[4] FAO, "Probiotics in food," Food Nutrition Paper, vol. 85, p. 71, 2001.

[5] C. Mantegazza, P. Molinari, E. D’Auria, M. Sonnino, L. Morelli, and G. V. Zuccotti, "Probiotics and antibioticassociated diarrhea in children: a review and new evidence on Lactobacillus rhamnosus GG during and after antibiotic treatment," Pharmacological Research, vol. 128, pp. 63-72, 2018.

[6] M. Amer, M. Nadeem, S. U. R. Nazir et al., "Probiotics and their use in inflammatory bowel disease," Alternative Therapies in Health and Medicine, vol. 24, no. 3, pp. 16-23, 2018.

[7] H. F. Dale, S. H. Rasmussen, Ö. Asiller, and G. A. Lied, "Probiotics in irritable bowel syndrome: an up-to-date systematic review," Nutrients, vol. 11, no. 9, p. 2048, 2019.

[8] M. Homan and R. Orel, "Are probiotics useful in Helicobacter pylori eradication?," World Journal of Gastroenterology, vol. 21, no. 37, pp. 10644-10653, 2015.

[9] M. de Vrese, A. Stegelmann, B. Richter, S. Fenselau, C. Laue, and J. Schrezenmeir, "Probiotics-compensation for lactase insufficiency," The American Journal of Clinical Nutrition, vol. 73, no. 2, pp. 421s-429s, 2001.

[10] M. C. Collado, M. Derrien, E. Isolauri, W. M. de Vos, and S. Salminen, "Intestinal integrity and Akkermansia muciniphila, a mucin-degrading member of the intestinal microbiota present in infants, adults, and the elderly," Applied and Environmental Microbiology, vol. 73, no. 23, pp. 7767-7770, 2007.

[11] B. Routy, E. le Chatelier, L. Derosa et al., "Gut microbiome influences efficacy of PD-1-based immunotherapy against epithelial tumors," Science, vol. 359, no. 6371, pp. 91-97, 2018.

[12] L. Shi, J. Sheng, G. Chen et al., "Combining IL-2-based immunotherapy with commensal probiotics produces enhanced antitumor immune response and tumor clearance," Journal for Immunotherapy of Cancer, vol. 8, no. 2, article e000973, 2020.

[13] B. Sánchez, C. G. D. L. Reyes-Gavilán, A. Margolles, and M. Gueimonde, "Probiotic fermented milks: present and future," International Journal of Dairy Technology, vol. 62, no. 4, pp. 472-483, 2009.

[14] A. Q. Yu and L. Li, "The potential role of probiotics in cancer prevention and treatment," Nutrition and Cancer, vol. 68, no. 4, pp. 535-544, 2016.

[15] B. Sánchez, S. Delgado, A. Blanco-Míguez, A. Lourenço, M. Gueimonde, and A. Margolles, "Probiotics, gut microbiota, and their influence on host health and disease," Molecular Nutrition \& Food Research, vol. 61, no. 1, 2017.

[16] C. L. Maynard, C. O. Elson, R. D. Hatton, and C. T. Weaver, "Reciprocal interactions of the intestinal microbiota and immune system," Nature, vol. 489, no. 7415, pp. 231-241, 2012.

[17] M. E. Johansson, J. M. Larsson, and G. C. Hansson, "The two mucus layers of colon are organized by the MUC2 mucin, whereas the outer layer is a legislator of host-microbial interactions," Proceedings of the National Academy of Sciences, vol. 108, Supplement_1, pp. 4659-4665, 2011.

[18] Y. Goto and I. I. Ivanov, "Intestinal epithelial cells as mediators of the commensal-host immune crosstalk," Immunology and Cell Biology, vol. 91, no. 3, pp. 204-214, 2013.

[19] S. Rakoff-Nahoum, J. Paglino, F. Eslami-Varzaneh, S. Edberg, and R. Medzhitov, "Recognition of commensal microflora by toll-like receptors is required for intestinal homeostasis," Cell, vol. 118, no. 2, pp. 229-241, 2004.

[20] S. Misra, D. Mohanty, and S. Mohapatra, "Applications of probiotics as a functional ingredient in food and gut health," Journal of Food and Nutrition Research, vol. 7, no. 3, pp. 213223, 2019. 
[21] C. M. Costello, R. M. Sorna, Y. L. Goh, I. Cengic, N. K. Jain, and J. C. March, "3-D intestinal scaffolds for evaluating the therapeutic potential of probiotics," Molecular Pharmaceutics, vol. 11, no. 7, pp. 2030-2039, 2014.

[22] Y. Lan, M. W. A. Verstegen, S. Tamminga, and B. A. Williams, "The role of the commensal gut microbial community in broiler chickens," World's Poultry ence Journal, vol. 61, no. 1, pp. 95-104, 2005.

[23] T. D. Lawley and A. W. Walker, "Intestinal colonization resistance," Immunology, vol. 138, no. 1, pp. 1-11, 2013.

[24] M. Wang, S. Wichienchot, X. He, X. Fu, Q. Huang, and B. Zhang, "In vitro colonic fermentation of dietary fibers: fermentation rate, short-chain fatty acid production and changes in microbiota," Trends in Food Science \& Technology, vol. 88, pp. 1-9, 2019.

[25] H. Brody, "Colorectal cancer," Nature, vol. 521, no. 7551, p. S1, 2015.

[26] K. Vipperla and S. J. O'Keefe, "Diet, microbiota, and dysbiosis: a 'recipe' for colorectal cancer," Food \& Function, vol. 7, no. 4, pp. 1731-1740, 2016.

[27] J. Björk, "Strategies for colon cancer prevention," The EPMA Journal, vol. 1, no. 3, pp. 513-521, 2010.

[28] O The Lancet, "Colorectal cancer: a disease of the young?," The Lancet Oncology, vol. 18, no. 4, p. 413, 2017.

[29] N. Barker, J. H. van Es, J. Kuipers et al., "Identification of stem cells in small intestine and colon by marker gene Lgr5," Nature, vol. 449, no. 7165, pp. 1003-1007, 2007.

[30] T. Sato, R. G. Vries, H. J. Snippert et al., "Single Lgr5 stem cells build crypt-villus structures in vitro without a mesenchymal niche," Nature, vol. 459, no. 7244, pp. 262-265, 2009.

[31] N. Barker, "Adult intestinal stem cells: critical drivers of epithelial homeostasis and regeneration," Nature Reviews. Molecular Cell Biology, vol. 15, no. 1, pp. 19-33, 2014.

[32] H. Clevers and E. Batlle, "SnapShot: the intestinal crypt," Cell, vol. 152, no. 5, pp. 1198-1198.e2, 2013.

[33] K. W. Kinzler, M. Nilbert, L. Su et al., "Identification of FAP locus genes from chromosome 5q21," Science, vol. 253, no. 5020, pp. 661-665, 1991.

[34] I. Nishisho, Y. Nakamura, Y. Miyoshi et al., "Mutations of chromosome 5q21 genes in FAP and colorectal cancer patients," Science, vol. 253, no. 5020, pp. 665-669, 1991.

[35] J. Drost, R. H. van Jaarsveld, B. Ponsioen et al., "Sequential cancer mutations in cultured human intestinal stem cells," Nature, vol. 521, no. 7550, pp. 43-47, 2015.

[36] M. Christie, R. N. Jorissen, D. Mouradov et al., "Different APC genotypes in proximal and distal sporadic colorectal cancers suggest distinct $\mathrm{WNT} / \beta$-catenin signalling thresholds for tumourigenesis," Oncogene, vol. 32, no. 39, pp. 4675-4682, 2013.

[37] M. Buchert, D. Athineos, H. E. Abud et al., "Genetic dissection of differential signaling threshold requirements for the Wnt/beta-catenin pathway in vivo," PLoS Genetics, vol. 6, no. 1, article e1000816, 2010.

[38] B. A. Methé, K. E. Nelson, M. Pop et al., "A framework for human microbiome research," Nature, vol. 486, no. 7402, pp. 215-221, 2012.

[39] E. Rinaldi, A. Consonni, E. Guidesi, M. Elli, R. Mantegazza, and F. Baggi, "Gut microbiota and probiotics: novel immune system modulators in myasthenia gravis?," Annals of the New York Academy of Sciences, vol. 1413, no. 1, pp. 49-58, 2018.
[40] E. B. Hollister, C. Gao, and J. Versalovic, "Compositional and functional features of the gastrointestinal microbiome and their effects on human health," Gastroenterology, vol. 146, no. 6, pp. 1449-1458, 2014.

[41] C. Huttenhower, D. Gevers, R. Knight et al., "Structure, function and diversity of the healthy human microbiome," Nature, vol. 486, no. 7402, pp. 207-214, 2012.

[42] K. R. Amato, C. J. Yeoman, G. Cerda et al., "Variable responses of human and non-human primate gut microbiomes to a Western diet," Microbiome, vol. 3, no. 1, p. 53, 2015.

[43] C. Greenhill, "gut microbiota, host genetics and diet interact to affect the risk of developing obesity and the metabolic syndrome," Nature Reviews. Endocrinology, vol. 11, no. 11, p. 630, 2015.

[44] C. de Filippo, D. Cavalieri, M. di Paola et al., "Impact of diet in shaping gut microbiota revealed by a comparative study in children from Europe and rural Africa," Proceedings of the National Academy of Sciences of the United States of America, vol. 107, no. 33, pp. 14691-14696, 2010.

[45] Q. Guo, F. Li, Y. Duan et al., "Oxidative stress, nutritional antioxidants and beyond," Science China. Life Sciences, vol. 63, no. 6, pp. 866-874, 2020.

[46] J. Yang and J. Yu, "The association of diet, gut microbiota and colorectal cancer: what we eat may imply what we get," Protein \& Cell, vol. 9, no. 5, pp. 474-487, 2018.

[47] S. J. O'Keefe, "Diet, microorganisms and their metabolites, and colon cancer," Nature Reviews. Gastroenterology \& Hepatology, vol. 13, no. 12, pp. 691-706, 2016.

[48] D. J. Morrison and T. Preston, "Formation of short chain fatty acids by the gut microbiota and their impact on human metabolism," Gut Microbes, vol. 7, no. 3, pp. 189-200, 2016.

[49] X. Wu, Y. Wu, L. He, L. Wu, X. Wang, and Z. Liu, "Effects of the intestinal microbial metabolite butyrate on the development of colorectal cancer," Journal of Cancer, vol. 9, no. 14, pp. 2510-2517, 2018.

[50] V. Tremaroli and F. Bäckhed, "Functional interactions between the gut microbiota and host metabolism," Nature, vol. 489, no. 7415, pp. 242-249, 2012.

[51] M. Song, W. S. Garrett, and A. T. Chan, "Nutrients, foods, and colorectal cancer prevention," Gastroenterology, vol. 148, no. 6, pp. 1244-1260.e16, 2015.

[52] M. Candela, S. Turroni, E. Biagi et al., "Inflammation and colorectal cancer, when microbiota-host mutualism breaks," World Journal of Gastroenterology, vol. 20, no. 4, pp. 908922, 2014.

[53] G. Nakatsu, X. Li, H. Zhou et al., "Gut mucosal microbiome across stages of colorectal carcinogenesis," Nature Communications, vol. 6 , no. 1, p. 8727, 2015.

[54] C. Yazici, P. G. Wolf, H. Kim et al., "Race-dependent association of sulfidogenic bacteria with colorectal cancer," Gut, vol. 66, no. 11, pp. 1983-1994, 2017.

[55] D. Lv, X. Xiong, H. Yang et al., "Effect of dietary soy oil, glucose, and glutamine on growth performance, amino acid profile, blood profile, immunity, and antioxidant capacity in weaned piglets," Science China. Life Sciences, vol. 61, no. 10, pp. 1233-1242, 2018.

[56] H. Tilg, T. E. Adolph, R. R. Gerner, and A. R. Moschen, "The intestinal microbiota in colorectal cancer," Cancer Cell, vol. 33, no. 6, pp. 954-964, 2018. 
[57] X. C. Morgan, N. Segata, and C. Huttenhower, "Biodiversity and functional genomics in the human microbiome," Trends in Genetics, vol. 29, no. 1, pp. 51-58, 2013.

[58] S. J. Bultman, "Interplay between diet, gut microbiota, epigenetic events, and colorectal cancer," Molecular Nutrition \& Food Research, vol. 61, no. 1, 2017.

[59] J. Rafter, "The effects of probiotics on colon cancer development," Nutrition Research Reviews, vol. 17, no. 2, pp. 277284, 2004.

[60] M. Eslami, B. Yousefi, P. Kokhaei et al., "Importance of probiotics in the prevention and treatment of colorectal cancer," Journal of Cellular Physiology, vol. 234, no. 10, pp. 1712717143, 2019.

[61] P. Louis, G. L. Hold, and H. J. Flint, "The gut microbiota, bacterial metabolites and colorectal cancer," Nature Reviews. Microbiology, vol. 12, no. 10, pp. 661-672, 2014.

[62] A. Alhenaky, A. Abdelqader, M. Abuajamieh, and A. R. AlFataftah, "The effect of heat stress on intestinal integrity and Salmonella invasion in broiler birds," Journal of Thermal Biology, vol. 70, no. Part B, pp. 9-14, 2017.

[63] D. Liu, X. Y. Jiang, L. S. Zhou, J. H. Song, and X. Zhang, "Effects of probiotics on intestinal mucosa barrier in patients with colorectal cancer after operation: meta-analysis of randomized controlled trials," Medicine (Baltimore), vol. 95, no. 15 , article e3342, 2016.

[64] C.-S. Alvarez, J. Badia, M. Bosch, R. Giménez, and L. Baldomà, "Outer membrane vesicles and soluble factors released by probiotic Escherichia coli Nissle 1917 and commensal ECOR63 enhance barrier function by regulating expression of tight junction proteins in intestinal epithelial cells," Frontiers in Microbiology, vol. 7, p. 1981, 2016.

[65] R. Martín, C. Chamignon, N. Mhedbi-Hajri et al., "The potential probiotic Lactobacillus rhamnosus CNCM I-3690 strain protects the intestinal barrier by stimulating both mucus production and cytoprotective response," Scientific Reports, vol. 9, no. 1, p. 5398, 2019.

[66] Y. Xu, L. Lahaye, Z. He, J. Zhang, C. Yang, and X. Piao, "Micro-encapsulated essential oils and organic acids combination improves intestinal barrier function, inflammatory responses and microbiota of weaned piglets challenged with enterotoxigenic Escherichia coli F4 (K88(+))," Animal Nutrition, vol. 6, no. 3, pp. 269-277, 2020.

[67] Y. Li, A. S. Fanning, J. M. Anderson, and A. Lavie, "Structure of the conserved cytoplasmic C-terminal domain of occludin: identification of the ZO-1 binding surface," Journal of Molecular Biology, vol. 352, no. 1, pp. 151-164, 2005.

[68] S. Guo, T. Gillingham, Y. Guo et al., "Secretions of Bifidobacterium infantis and Lactobacillus acidophilus protect intestinal epithelial barrier function," Journal of Pediatric Gastroenterology and Nutrition, vol. 64, no. 3, pp. 404-412, 2017.

[69] J. Wang, H. Ji, S. Wang et al., "Probiotic Lactobacillus plantarum promotes intestinal barrier function by strengthening the epithelium and modulating gut microbiota," Frontiers in Microbiology, vol. 9, p. 1953, 2018.

[70] M. E. Johansson, M. Phillipson, J. Petersson, A. Velcich, L. Holm, and G. C. Hansson, "The inner of the two Muc2 mucin-dependent mucus layers in colon is devoid of bacteria," Proceedings of the National Academy of Sciences of the United States of America, vol. 105, no. 39, pp. 1506415069, 2008.
[71] M. van der Sluis, B. A. E. de Koning, A. C. J. M. de Bruijn et al., "Muc2-deficient mice spontaneously develop colitis, indicating that MUC2 is critical for colonic protection," Gastroenterology, vol. 131, no. 1, pp. 117-129, 2006.

[72] W. Fong, Q. Li, and J. Yu, "Gut microbiota modulation: a novel strategy for prevention and treatment of colorectal cancer," Oncogene, vol. 39, no. 26, pp. 4925-4943, 2020.

[73] C. A. Brennan and W. S. Garrett, "Gut microbiota, inflammation, and colorectal cancer," Annual Review of Microbiology, vol. 70, no. 1, pp. 395-411, 2016.

[74] H. Tlaskalova-Hogenova, L. Vannucci, K. Klimesova, R. Stepankova, J. Krizan, and M. Kverka, "Microbiome and colorectal carcinoma: insights from germ-free and conventional animal models," Cancer Journal, vol. 20, no. 3, pp. 217-224, 2014.

[75] Y. H. Ding, L. Y. Qian, J. Pang et al., "The regulation of immune cells by lactobacilli: a potential therapeutic target for anti-atherosclerosis therapy," Oncotarget, vol. 8, no. 35, pp. 59915-59928, 2017.

[76] M. Molska and J. Reguła, "Potential mechanisms of probiotics action in the prevention and treatment of colorectal cancer," Nutrients, vol. 11, no. 10, p. 2453, 2019.

[77] S. H. Lee, P. M. Starkey, and S. Gordon, "Quantitative analysis of total macrophage content in adult mouse tissues. Immunochemical studies with monoclonal antibody F4/80," The Journal of Experimental Medicine, vol. 161, no. 3, pp. 475-489, 1985.

[78] A. M. Platt, C. C. Bain, Y. Bordon, D. P. Sester, and A. M. I. Mowat, "An independent subset of TLR expressing CCR2-dependent macrophages promotes colonic inflammation," Journal of Immunology, vol. 184, no. 12, pp. 68436854, 2010.

[79] E. Zigmond, C. Varol, J. Farache et al., "Ly6C hi monocytes in the inflamed colon give rise to proinflammatory effector cells and migratory antigen-presenting cells," Immunity, vol. 37, no. 6, pp. 1076-1090, 2012.

[80] R. A. Isidro and C. B. Appleyard, "Colonic macrophage polarization in homeostasis, inflammation, and cancer," American Journal of Physiology. Gastrointestinal and Liver Physiology, vol. 311, no. 1, pp. G59-G73, 2016.

[81] H. J. Wu and E. Wu, "The role of gut microbiota in immune homeostasis and autoimmunity," Gut Microbes, vol. 3, no. 1, pp. 4-14, 2014.

[82] I. C. Chung, C. N. OuYang, S. N. Yuan et al., "Pretreatment with a heat-killed probiotic modulates the NLRP3 inflammasome and attenuates colitis-associated colorectal cancer in mice," Nutrients, vol. 11, no. 3, p. 516, 2019.

[83] M. Kasubuchi, S. Hasegawa, T. Hiramatsu, A. Ichimura, and I. Kimura, "Dietary gut microbial metabolites, short-chain fatty acids, and host metabolic regulation," Nutrients, vol. 7, no. 4, pp. 2839-2849, 2015.

[84] N. Singh, A. Gurav, S. Sivaprakasam et al., "Activation of Gpr109a, receptor for niacin and the commensal metabolite butyrate, suppresses colonic inflammation and carcinogenesis," Immunity, vol. 40, no. 1, pp. 128-139, 2014.

[85] M. Perše, "Oxidative stress in the pathogenesis of colorectal cancer: cause or consequence?," BioMed Research International, vol. 2013, Article ID 725710, 9 pages, 2013.

[86] M. L. Circu and T. Y. Aw, "Intestinal redox biology and oxidative stress," Seminars in Cell \& Developmental Biology, vol. 23, no. 7, pp. 729-737, 2012. 
[87] N. Mach and D. Fuster-Botella, "Endurance exercise and gut microbiota: a review," Journal of Sport and Health Science, vol. 6, no. 2, pp. 179-197, 2017.

[88] G. Guan, S. Ding, Y. Yin, V. Duraipandiyan, N. A. al-Dhabi, and G. Liu, "Macleaya cordata extract alleviated oxidative stress and altered innate immune response in mice challenged with enterotoxigenic Escherichia coli," Science China. Life Sciences, vol. 62, no. 8, pp. 1019-1027, 2019.

[89] K. Richter and T. Kietzmann, "Reactive oxygen species and fibrosis: further evidence of a significant liaison," Cell and Tissue Research, vol. 365, no. 3, pp. 591-605, 2016.

[90] I. Amrouche-Mekkioui and B. Djerdjouri, "N-acetylcysteine improves redox status, mitochondrial dysfunction, mucindepleted crypts and epithelial hyperplasia in dextran sulfate sodium-induced oxidative colitis in mice," European Journal of Pharmacology, vol. 691, no. 1-3, pp. 209-217, 2012.

[91] W. Tang, J. Wu, S. Jin et al., "Glutamate and aspartate alleviate testicular/epididymal oxidative stress by supporting antioxidant enzymes and immune defense systems in boars," Science China. Life Sciences, vol. 63, no. 1, pp. 116-124, 2020.

[92] X. Hua, A. I. Phipps, A. N. Burnett-Hartman et al., "Timing of aspirin and other nonsteroidal anti-inflammatory drug use among patients with colorectal cancer in relation to tumor markers and survival," Journal of Clinical Oncology, vol. 35, no. 24, pp. 2806-2813, 2017.

[93] G. J. Tsioulias, M. F. Go, and B. Rigas, "NSAIDs and colorectal cancer control: promise and challenges," Current Pharmacology Reports, vol. 1, no. 5, pp. 295-301, 2015.

[94] J. L. Arbiser, J. Petros, R. Klafter et al., "Reactive oxygen generated by Nox1 triggers the angiogenic switch," Proceedings of the National Academy of Sciences of the United States of America, vol. 99, no. 2, pp. 715-720, 2002.

[95] K. Rokutan, T. Kawahara, Y. Kuwano, K. Tominaga, A. Sekiyama, and S. Teshima-Kondo, "NADPH oxidases in the gastrointestinal tract: a potential role of Noxl in innate immune response and carcinogenesis," Antioxidants \& Redox Signaling, vol. 8, no. 9-10, pp. 1573-1582, 2006.

[96] A. Mangerich, P. C. Dedon, J. G. Fox, S. R. Tannenbaum, and G. N. Wogan, "Chemistry meets biology in colitis-associated carcinogenesis," Free Radical Research, vol. 47, no. 11, pp. 958-986, 2013.

[97] M. E. Hope, G. L. Hold, R. Kain, and E. M. El-Omar, "Sporadic colorectal cancer-role of the commensal microbiota," FEMS Microbiology Letters, vol. 244, no. 1, pp. 1-7, 2005.

[98] A. C. Goodwin, C. E. D. Shields, S. Wu et al., "Polyamine catabolism contributes to enterotoxigenic Bacteroides fragilis-induced colon tumorigenesis," Proceedings of the National Academy of Sciences of the United States of America, vol. 108, no. 37, pp. 15354-15359, 2011.

[99] S. H. Ko, J. I. Jeon, H. A. Woo, and J. M. Kim, "Bacteroides fragilisenterotoxin upregulates heme oxygenase- 1 in dendritic cellsviareactive oxygen species-, mitogen-activated protein kinase-, and Nrf2-dependent pathway," World Journal of Gastroenterology, vol. 26, no. 3, pp. 291-306, 2020.

[100] A. Kumar, H. Wu, L. S. Collier-Hyams et al., "Commensal bacteria modulate cullin-dependent signaling via generation of reactive oxygen species," The EMBO Journal, vol. 26, no. 21, pp. 4457-4466, 2007.

[101] J. Xu, C. Xu, X. Chen et al., "Regulation of an antioxidant blend on intestinal redox status and major microbiota in early weaned piglets," Nutrition, vol. 30, no. 5, pp. 584-589, 2014.
[102] S. Ghosh, C. Dai, K. Brown et al., "Colonic microbiota alters host susceptibility to infectious colitis by modulating inflammation, redox status, and ion transporter gene expression," American Journal of Physiology. Gastrointestinal and Liver Physiology, vol. 301, no. 1, pp. G39-G49, 2011.

[103] M. Liu, W. Xie, X. Wan, and T. Deng, "Clostridium butyricum modulates gut microbiota and reduces colitis associated colon cancer in mice," International Immunopharmacology, vol. 88, p. 106862, 2020.

[104] K. B. Arun, A. Madhavan, T. R. Reshmitha, S. Thomas, and P. Nisha, "Short chain fatty acids enriched fermentation metabolites of soluble dietary fibre from Musa paradisiaca drives HT29 colon cancer cells to apoptosis," PLoS ONE, vol. 14, no. 5, article e0216604, 2019.

[105] P. Hu, W. Song, Y. Shan et al., "Lactobacillus paracasei subsp. paracasei M5L induces cell cycle arrest and calreticulin translocation via the generation of reactive oxygen species in HT29 cell apoptosis," Food \& Function, vol. 6, no. 7, pp. 22572265, 2015.

[106] Y. Zhang and Y. Li, "Engineering the antioxidative properties of lactic acid bacteria for improving its robustness," Current Opinion in Biotechnology, vol. 24, no. 2, pp. 142-147, 2013.

[107] M. Linsalata, A. Cavallini, C. Messa, A. Orlando, M. Refolo, and F. Russo, "Lactobacillus rhamnosus GG influences polyamine metabolism in HGC-27 gastric cancer cell line: a strategy toward nutritional approach to chemoprevention of gastric cancer," Current Pharmaceutical Design, vol. 16, no. 7, pp. 847-853, 2010.

[108] K. Dev, N. A. Mir, A. Biswas et al., "Dietary synbiotic supplementation improves the growth performance, body antioxidant pool, serum biochemistry, meat quality, and lipid oxidative stability in broiler chickens," Animal Nutrition, vol. 6, no. 3, pp. 325-332, 2020.

[109] I. Wollowski, G. Rechkemmer, and B. L. Pool-Zobel, "Protective role of probiotics and prebiotics in colon cancer," The American Journal of Clinical Nutrition, vol. 73, no. 2, pp. 451s-455s, 2001.

[110] H. Tjalsma, A. Boleij, J. R. Marchesi, and B. E. Dutilh, “A bacterial driver-passenger model for colorectal cancer: beyond the usual suspects," Nature Reviews. Microbiology, vol. 10, no. 8, pp. 575-582, 2012.

[111] K. M. Tuohy, G. C. Rouzaud, W. M. Brück, and G. R. Gibson, "Modulation of the human gut microflora towards improved health using prebiotics-assessment of efficacy," Current Pharmaceutical Design, vol. 11, no. 1, pp. 75-90, 2005.

[112] J. P. Nougayrède, S. Homburg, F. Taieb et al., "Escherichia coli induces DNA double-strand breaks in eukaryotic cells," Science, vol. 313, no. 5788, pp. 848-851, 2006.

[113] G. Cuevas-Ramos, C. R. Petit, I. Marcq, M. Boury, E. Oswald, and J. P. Nougayrede, "Escherichia coli induces DNA damage in vivo and triggers genomic instability in mammalian cells," Proceedings of the National Academy of Sciences of the United States of America, vol. 107, no. 25, pp. 11537-11542, 2010.

[114] N. Ulger Toprak, A. Yagci, B. M. Gulluoglu et al., "A possible role of Bacteroides fragilis enterotoxin in the aetiology of colorectal cancer," Clinical Microbiology and Infection, vol. 12, no. 8, pp. 782-786, 2006.

[115] A. Verma and G. Shukla, "Probiotics Lactobacillus rhamnosus GG, Lactobacillus acidophilus suppresses DMH-induced procarcinogenic fecal enzymes and preneoplastic aberrant crypt foci in early colon carcinogenesis in Sprague Dawley rats," Nutrition and Cancer, vol. 65, no. 1, pp. 84-91, 2013. 
[116] A. Nowak, K. Śliżewska, J. Błasiak, and Z. Libudzisz, "The influence of Lactobacillus casei DN 114001 on the activity of faecal enzymes and genotoxicity of faecal water in the presence of heterocyclic aromatic amines," Anaerobe, vol. 30, pp. 129-136, 2014.

[117] C. L. Vernazza, B. A. Rabiu, and G. R. Gibson, "Human Colonic Microbiology and the Role of Dietary Intervention: Introduction to Prebiotic," in Prebiotics: Development \& Application, John Wiley \& Sons, Ltd, 2012.

[118] A. de Moreno de LeBlanc and G. Perdigón, "Reduction of $\beta$ Glucuronidase and nitroreductase activity by yoghurt in a murine colon cancer model," Biocell, vol. 29, no. 1, pp. 1524, 2005.

[119] P. Marteau, P. Pochart, B. Flourié et al., "Effect of chronic ingestion of a fermented dairy product containing Lactobacillus acidophilus and Bifidobacterium bifidum on metabolic activities of the colonic flora in humans," The American Journal of Clinical Nutrition, vol. 52, no. 4, pp. 685-688, 1990.

[120] D. Goossens, D. Jonkers, M. Russel, E. Stobberingh, A. van den Bogaard, and R. StockbrÜgger, "The effect of Lactobacillus plantarum $299 \mathrm{v}$ on the bacterial composition and metabolic activity in faeces of healthy volunteers: a placebocontrolled study on the onset and duration of effects," Alimentary Pharmacology \& Therapeutics, vol. 18, no. 5, pp. 495-505, 2003.

[121] S. Elmore, "Apoptosis: a review of programmed cell death10.1080/01926230701320337," Toxicologic Pathology, vol. 35, no. 4, pp. 495-516, 2007.

[122] S. W. Fesik, "Promoting apoptosis as a strategy for cancer drug discovery," Nature Reviews. Cancer, vol. 5, no. 11, pp. 876-885, 2005.

[123] Y. Gamallat, A. Meyiah, E. D. Kuugbee et al., "Lactobacillus rhamnosus induced epithelial cell apoptosis, ameliorates inflammation and prevents colon cancer development in an animal model," Biomedicine \& Pharmacotherapy, vol. 83, pp. 536-541, 2016.

[124] J. Y. Zhang, X. Xiao, Y. Dong, J. Wu, F. Yao, and X. H. Zhou, "Effect of fermented wheat germ extract with Lactobacillus plantarum dy-1 on HT-29 cell proliferation and apoptosis," Journal of Agricultural and Food Chemistry, vol. 63, no. 9, pp. 2449-2457, 2015.

[125] C. Avivi-Green, S. Polak-Charcon, Z. Madar, and B. Schwartz, "Apoptosis cascade proteins are regulated in vivo by high intracolonic butyrate concentration: correlation with colon cancer inhibition," Oncology Research, vol. 12, no. 2, pp. 83-95, 2000.

[126] A. Lan, D. Lagadic-Gossmann, C. Lemaire, C. Brenner, and G. Jan, "Acidic extracellular pH shifts colorectal cancer cell death from apoptosis to necrosis upon exposure to propionate and acetate, major end-products of the human probiotic propionibacteria," Apoptosis, vol. 12, no. 3, pp. 573-591, 2007.

[127] L. Pattayil and H. T. Balakrishnan-Saraswathi, "In vitroevaluation of apoptotic induction of butyric acid derivatives in colorectal carcinoma cells," Anticancer Research, vol. 39, no. 7, pp. 3795-3801, 2019.

[128] M. Comalada, E. Bailón, O. de Haro et al., "The effects of short-chain fatty acids on colon epithelial proliferation and survival depend on the cellular phenotype," Journal of Cancer Research and Clinical Oncology, vol. 132, no. 8, pp. 487-497, 2006.
[129] N. Mustapha, A. Pinon, Y. Limami et al., "Crataegus azarolus leaves induce antiproliferative activity, cell cycle arrest, and apoptosis in human HT-29 and HCT-116 colorectal cancer cells," Journal of Cellular Biochemistry, vol. 117, no. 5, pp. 1262-1272, 2016.

[130] A. Bharwani, M. F. Mian, M. G. Surette, J. Bienenstock, and P. Forsythe, "Oral treatment with Lactobacillus rhamnosus attenuates behavioural deficits and immune changes in chronic social stress," BMC Medicine, vol. 15, no. 1, p. 7, 2017.

[131] A. Quagliariello, I. Aloisio, N. Bozzi Cionci et al., "Effect of Bifidobacterium breve on the intestinal microbiota of coeliac children on a gluten free diet: a pilot study," Nutrients, vol. 8, no. 10, p. 660, 2016.

[132] T. S. Manning and G. R. Gibson, "Microbial-gut interactions in health and disease. Prebiotics," Best Practice \& Research. Clinical Gastroenterology, vol. 18, no. 2, pp. 287-298, 2004.

[133] J. Denizot, A. Desrichard, A. Agus et al., "Diet-induced hypoxia responsive element demethylation increases CEACAM6 expression, favouring Crohn's disease-associated Escherichia coli colonisation," Gut, vol. 64, no. 3, pp. 428-437, 2015.

[134] M. Bonnet, E. Buc, P. Sauvanet et al., "Colonization of the human gut by E. coli and colorectal cancer risk," Clinical Cancer Research, vol. 20, no. 4, pp. 859-867, 2014.

[135] J. C. Arthur, R. Z. Gharaibeh, M. Mühlbauer et al., "Microbial genomic analysis reveals the essential role of inflammation in bacteria-induced colorectal cancer," Nature Communications, vol. 5, no. 1, p. 4724, 2014.

[136] E. L. Ma, Y. J. Choi, J. Choi, C. Pothoulakis, S. H. Rhee, and E. Im, "The anticancer effect of probioticBacillus polyfermenticuson human colon cancer cells is mediated through ErbB2 and ErbB3 inhibition," International Journal of Cancer, vol. 127, no. 4, article NA-790, 2010.

[137] C. G. Olnood, S. S. M. Beski, P. A. Iji, and M. Choct, "Delivery routes for probiotics: effects on broiler performance, intestinal morphology and gut microflora," Animal Nutrition, vol. 1, no. 3, pp. 192-202, 2015.

[138] K. Saito, S. Koido, T. Odamaki et al., "Metagenomic analyses of the gut microbiota associated with colorectal adenoma," PLoS ONE, vol. 14, no. 2, article e0212406, 2019.

[139] J. Rafter, M. Bennett, G. Caderni et al., "Dietary synbiotics reduce cancer risk factors in polypectomized and colon cancer patients," The American Journal of Clinical Nutrition, vol. 85, no. 2, pp. 488-496, 2007. 